\title{
Use of the frailty tools FI MDS and FI Rockwood to predict outcomes of inpatient rehabilitation after proximal hip fracture
}

Oxana Zalyesov, Muhamad Badarny, Natalia Zaygraykin, Jochanan E. Naschitz*

Bait Balev Nesher, Department of Rehabilitation

*Corresponding Author: Jochanan E. Naschitz, POB 252, Nesher 36602, Derech Hashalom 11. Telephone 073-2-377-300, Fax 97248345575.

Received date: June 07, 2021; Accepted date: June 28, 2021; Published date: July 02, 2021

Citation: O Zalyesov, M Badarny, N Zaygraykin, J E. Naschitz. (2021) Use of the frailty tools FI MDS and FI Rockwood to predict outcomes of inpatient rehabilitation after proximal hip fracture. Clinical Research and Clinical Trials 4(1); DOI: 10.31579/2693-4779/049

Copyright: (C) 2021 Jochanan E. Naschitz. This is an open access article distributed under the Creative Commons Attribution License, which permits unrestricted use, distribution, and reproduction in any medium, provided the original work is properly cited.

\begin{abstract}
Background: Among factors which affect the outcome of rehabilitation after hip fracture, the role of pre-fracture frailty has not been defined.

Objective: To assess the impact of frailty on rehabilitation outcome in elderly persons with proximal hip fracture.

Setting: Geriatric rehabilitation center.

Design: Retrospective observational study.

Subjects: Forty consecutive patients with proximal hip fracture.

Methods: The following parameters were calculated: FI-MDS frailty index, Rockwood Clinical Frailty Scale (FI Rockwood), Functional Independence Measure (FIM) on discharge, absolute functional gain ( $\triangle$ FIM), length of stay (LOS), and FIM gain per day ( $\triangle$ FIM/LOS). FIM discharge $\geq 90$ and motor FIM discharge $>58$ indicated success in rehabilitation.

Results: There were 31 women and 9 men, mean age 80 years. The mean pre-fracture FI MDS was 10 points (SD 5.8) out of 58 possible, the mean FI Rockwood score was 3 (SD 1.7) out of 7 possible. The mean admission FIM was 76.6 (SD 16), the mean discharge FIM was 92.7 (SD 19.3), mean LOS 22.5 days (SD 9.7), mean $\triangle$ FIM/LOS 0.8 (SD 0.6). Pre-fracture frailty was associated with worse outcome of rehabilitation: FI MDS predicted discharge FIM $\geq 90$ with OR 0.86, p 0.012; FI Rockwood predicted discharge FIM $\geq 90$ with OR 0.68, p 0.027; FI MDS predicted discharge motor FIM >58 with OR 0.91, p 0.07; FI Rockwood predicted discharge motor FIM >58 with OR 0.71, p 0.027.
\end{abstract}

Conclusions: Though pre-fracture frailty correlated with FIM on discharge, neither frailty tool was specific enough to predict success in rehabilitation and the necessary length of rehabilitation. Translating frailty scores in tools adequate for clinical decision making remains an aim for future studies.

Keywords: frailty; hip fracture; rehabilitation; functional independence measure (FIM)

\section{Introduction}

Numerous factors affect the outcome of rehabilitation in patients with hip fractures, including the patient's age, gender, pre-fracture functional ability, cognitive condition, affective status, comorbidity, social support, and the number of treatments [1-5]. The outcome of inpatient rehabilitation after hip fracture surgery may well be affected by frailty that pre-existed to hip fracture. It is assumed that frailty tools may help in guiding management decisions in patient care [6-13].

Frailty is understood as the increased vulnerability to stressors and limited homeostatic reserves to stabilize declines across multiple physiologic systems. Adults who are frail are prone to developing disease and have worse disease outcomes. Frailty that predicts greater clinical risk [14]. No single frailty assessment tool has become dominant. Fried LP et al. labeled an operational phenotype of frailty. Accordingly, frailty is a clinical syndrome in which three or more of the following alterations are present: unintentional weight loss ( $4.5 \mathrm{~kg}$ in the past year), self-reported exhaustion, weakness (by grip strength measurement), slow walking speed, and low level of physical activity [15]. Many other frailty scores have been proposed [16-20]. Ideally a frailty tool should be quick, reliable, and easy to use in clinical settings. These demands are not met by most frailty tools which are in common use but are well served by two 
instruments: the FI-MDS frailty index [21] and the Rockwood Clinical Frailty Scale [22]. The two latter do not require examination of physical performance nor the need of patients' self-reporting their impairments. The FI MDS and FI Rockwood are informed by cognitive, motor and social functioning, disability and morbidities, influenced by psychological factors and social support. Thus, the frailty score is the equivalent of a battery of tests. The FI MDS and FI Rockwood are both appropriate for use in subjects with physical impairment and cognitive decline, features often met in older subjects recovering from hip fracture.

In the present study we assessed whether pre-fracture frailty affected the outcome of rehabilitation in older persons with proximal hip fracture. The FI-MDS frailty index and the Rockwood Clinical Frailty Scale were used as comprehensive measures of the patients' health status before fracture. The Functional Independence Measure (FIM) on discharge served as a measure of rehabilitation outcome.

\section{Methods}

The Institutional Review Board approved this retrospective observational study and waived the need for obtaining patients' informed consent. The study was conducted in a 40- bed rehabilitation department that admits mainly orthopedic patients. Included were consecutive patients aged 65 years or older transferred from orthopedic surgery to the geriatric rehabilitation ward. Excluded were non-cooperative subjects, those having an infected operation site, and those temporarily prohibited to tread. The following study parameters were appraised, each-one by independent observers: the FIM at the time of admission, FIM at the time of discharge, the absolute functional gain (calculated as the difference between FIM discharge and FIM admission, i.e. $\Delta$ FIM), the daily gain of FIM (calculated by dividing the absolute functional gain by the length of stay in rehabilitation, i.e. $\Delta$ FIM/LOS), the Rockwood Clinical Frailty scale (FI Rockwood), the frailty index calculated from the Minimum Data Set document (FI-MDS), the Mini-Mental State Examination of Folstein (MMSE), and the length of stay in rehabilitation (LOS).

Pre-fracture frailty was assessed by physicians who were directly involved in the patients' care. Two frailty tools were used, the FIRockwood [22] and FI-MDS, the latter was calculated from the Minimum Data Set document [23]. From the MDS document a list of 58 deficits was derived representing multiple functional domains. Each deficit was assigned either 0 (absence of the condition or attribute) or 1 (presence of the condition or attribute). The body mass index was assigned score 0 unless it was $<18.5 \mathrm{~kg} / \mathrm{m} 2$ or $\geq 30 \mathrm{~kg} / \mathrm{m} 2$, in which case a score of 1 was assigned. Medication usage was scored according to the number of medications used: $6-9$ score $1 ; 10-14$ score $2 ; 15-19$ score $3 ;>20$ score 4 . The mean of the deficits was expressed as a proportion of the total 58 points, ranging from 0 (no deficits) to $100 \%$ (58 deficits) [23]. By the Rockwood Clinical Frailty Scale the examiner's intuitive perception of a patient's disability and cognitive impairment was represented by a score ranging from 1 (robust health) to 7 (complete functional dependence) [22]. The Mini-Mental State Examination (MMSE) of Folstein [24] was used assess the patients' cognitive state at the time of admission.

The Functional Independence Measure (FIM) is used in rehabilitation to explore an individual's physical, psychological, and social functions and to monitor the progress under rehabilitation. The FIM has two subscales: the motor subscale, consisting of 13 items related to self-care, transfers, and locomotion, and the cognitive subscale, consisting of 5 items related to comprehension, expression, and memory. Each item is assigned a rating of 1-7, where 1 denotes the necessity for assistance and 7 denotes complete independence [25]. The 'FIM admission' is administered within 3 days of admission to rehabilitation and the 'FIM discharge' within 3 days before discharge by the same team. According to common knowledge, total FIM on discharge $\geq 90$ or motor FIM on discharge $>58$ signify that a person can be discharged home [6].

Rehabilitation involved the diagnosis of a person's problems and needs, defining rehabilitation goals, and therapeutic interventions in order to regain maximum self-sufficiency. Rehabilitation was provided by a multidisciplinary team consisting of physicians, occupational therapists, physical therapists, social workers, speech and language therapists, dietitians, and nurses. The patients received 5 days per week standard physical therapy (i.e., walking, climbing stairs, balance, muscle strength, and range of motion) and occupational therapy (i.e., basic ADL, instrumental ADL, and environment advice). The outcome of rehabilitation was evaluated by three measures: 1 ). the absolute functional gain from admission to discharge, i.e. $\Delta$ FIM, 2). FIM discharge $\geq 90$, and 3) motor FIM discharge $>58$.

We assessed which degree of pre-fracture frailty is compatible with rehabilitation success, the latter corresponding to FIM discharge $\geq 90$ or motor FIM discharge $>58$. Statistical analysis by MedCalc ${ }^{\circledR}$ Statistical Software version 19.8 (MedCalc Software Ltd, Ostend, Belgium; https://www.medcalc.org; 2021) used descriptive statistics, Pearson's correlation, Receiver Operating Characteristic curve, and logistic regression analysis, as appropriate. $\mathrm{P}<0.05$ was considered significant.

No funding was provided for this study.

\section{Results}

The data of 45 consecutive patients who met the inclusion criteria were reviewed. Five patients could not complete rehabilitation because of intercurrent illness needing hospitalization and were excluded from the analysis. Among the remaining 40 subjects there were 31 women and 9 men, their mean age was 80 years. The FI scores, MMSE, FIM on admission, FIM on discharge, motor FIM on discharge, $\triangle F I M$, LOS and $\Delta$ FIM/LOS are shown in Table 1. The mean $\triangle$ FIM (FIM improvement from admission to discharge) was 16.6 points (SD 9.9). The mean functional gain was of 0.8 FIM points per day (SD 0.6). 


\begin{tabular}{|l|l|l|l|}
\hline Variables & Mean & SD & Range \\
\hline Age (years) & 80 & 9 & \\
\hline FI MDS (\% out of 58 variables) & 10 & 5.8 & $2-31$ \\
\hline FI Rockwood (scale 1-7) & 3 & 1.7 & $1-6$ \\
\hline MMSE & 22 & 7 & $8-30$ \\
\hline FIM admission & 71.5 & 15 & $36-106$ \\
\hline FIM admission $\geq 90$ & No: $: 5$ & & \\
\hline FIM discharge & 91.4 & 10.1 & $40-115$ \\
\hline$\Delta$ FIM & 16.6 & 9.9 & $0-47$ \\
\hline FIM discharge $\geq 90$ & No: 20 & & \\
\hline Motor FIM discharge & 65 & 15.5 & $12-81$ \\
\hline LOS (days) & 22.5 & 9.7 & $9-60$ \\
\hline$\Delta$ FIMLOS & 0.8 & 0.6 & $-0.3-2.6$ \\
\hline
\end{tabular}

Table 1. Patient characteristics and outcomes of rehabilitation. FI: frailty index; MMSE: mini-mental state examination; FIM: functional independence measure; $\triangle F I M=$ FIM discharge - FIM admission; LOS: length of stay; $\triangle F I M / L O S:$ FIM gain per day.

The correlations between pre-fracture frailty by either method and FIM at the time of discharge are shown in Figure 1. Also represented in this figure are the pace of FIM improvement under rehabilitation (delta FIM / LOS) and the correlation between discharge FIM and LOS. It is noticed that a longer LOS compensated for a slower pace of improvement as to attain greater FIM at the time of discharge.
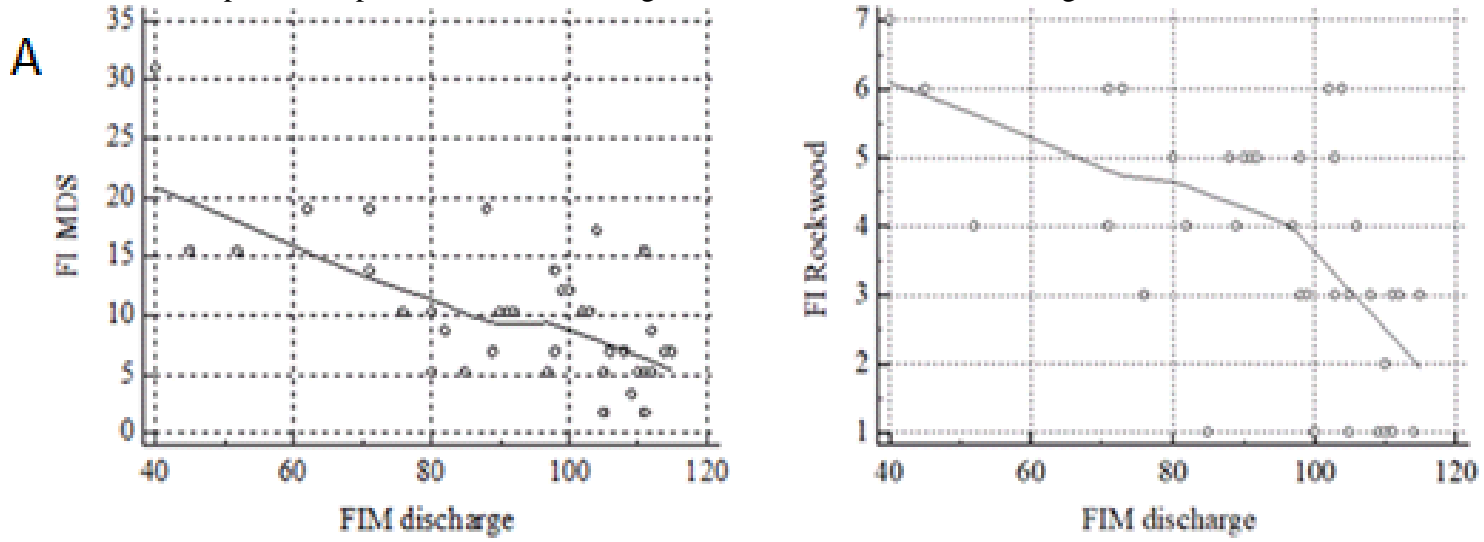

B
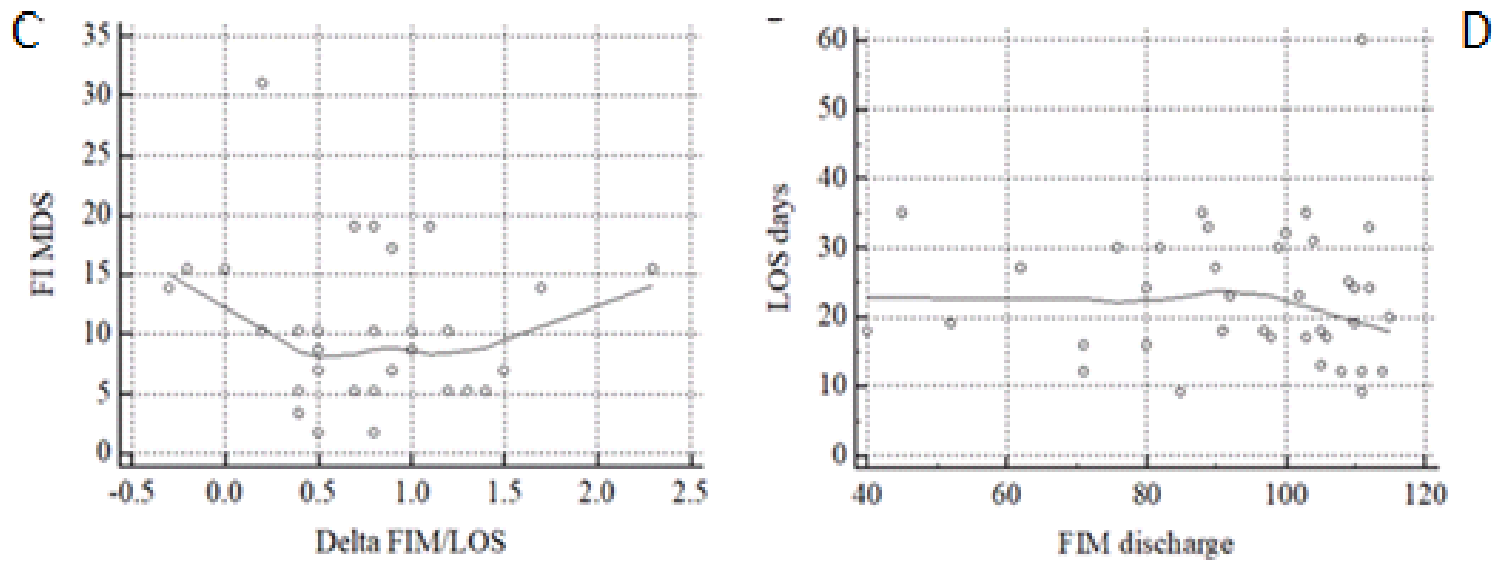

Figure 1. A. Correlation between the pre-fracture FI MDS and FIM discharge: $R-0.67, p<0.0001$. B. Correlation between pre-fracture FI Rockwood and FIM discharge: $R-0.58, p$ 0.0001. C. Correlation between pre-fracture FI MDS and $\triangle F I M / L O S$ (daily FIM gain): $R-0.15, p 0.35 . D$. Correlation between LOS and FIM discharge: $R-0.016, p 0.9$. 
Alike FI MDS, FI Rockwood correlated poorly with $\Delta$ FIM/LOS: R -0.15, p 0.35 .

Two measures serve as surrogate indicators that a patient is fit to be discharged home: a total FIM discharge $\geq 90$ and a motor FIM discharge $>58$ (17). In the present cohort, five patients scored FIM $\geq 90$ on admission; their number increased to 20 at the time of discharge.

Logistic regression analysis showed that pre-fracture frailty, by FI MDS as well as by FI Rockwood, is associated with and determines success in rehabilitation (Table 2).

\begin{tabular}{|l|l|l|}
\hline Frailty measures predicting success in rehabilitation & OR & P value \\
\hline FI MDS vs. FIM discharge $\geq 90$ & 0.86 & 0.012 \\
\hline FI MDS vs. motor FIM discharge $>58$ & 0.91 & 0.07 \\
\hline FI Rockwolod vs. FIM discharge $\geq 90$ & 0.68 & 0.027 \\
\hline FI Rockwood vs. motor FIM discharge $>$ 58 & 0.71 & 0.029 \\
\hline
\end{tabular}

Table 2. Pre-fracture frailty predicting success in rehabilitation.

By means of receiver operating characteristic (ROC) curves the performance of pre-fracture frailty scores in achieving determined FIM discharge thresholds were analyzed. The data is shown in Table 3 and Figure 2.

\begin{tabular}{|l|l|l|l|l|l|l|l|}
\hline $\begin{array}{l}\text { Defined FIM } \\
\text { threshold }\end{array}$ & Variable & AUC & $95 \% \mathrm{Cl}$ & $\mathrm{p}$ & Criterion & Sensibility \% & Specificity \% \\
\hline $\begin{array}{l}\text { Total FIM discharge } \\
\geq 90\end{array}$ & FI MDS & 0.74 & $0.57-0.86$ & 0.007 & $\leq 12.1$ & 88.5 & 53.8 \\
\hline $\begin{array}{l}\text { Total FIM discharge } \\
\geq 90\end{array}$ & FI Rockwood & 0.75 & $0.58-0.87$ & 0.0034 & $\leq 4$ & 73 & 53.8 \\
\hline $\begin{array}{l}\text { Motor FIM } \\
\text { discharge }>58\end{array}$ & FI MDS & 0.73 & $0.56-0.86$ & 0.019 & $\leq 12.1$ & 85.7 & 54.4 \\
\hline $\begin{array}{l}\text { Motor FIM } \\
\text { discharge }>58\end{array}$ & FI Rockwood & 0.73 & $0.56-0.86$ & 0.013 & $\leq 4$ & 71.4 & 54.5 \\
\hline
\end{tabular}

Table 3. Sensitivity and specificity of pre-fracture FI MDS and FI Rockwood for defined FIM outcomes, based on ROC curve analysis.

For total FIM discharge $\geq 90$, pre-fracture FI MDS best cutoff was $\leq 12.1$, sensitivity $88.5 \%$ and specificity $53.8 \%$; pre-fracture FI Rockwood best cutoff was $\leq 4$, sensitivity $73 \%$ and specificity $53.8 \%$. Similar results were observed for motor FIM discharge $>58$. Dose response plots provide

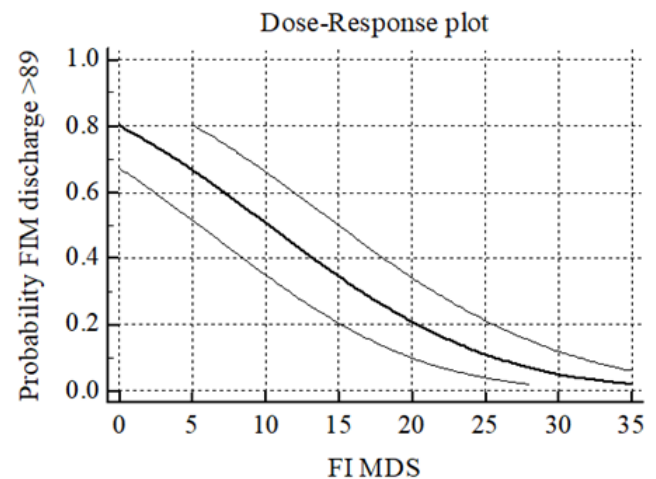

graphical illustration of frailty score-related probability that patients will reach desired total FIM threshold at the time of discharge. Similar constructs were seen for motor FIM discharge >58 (not shown).

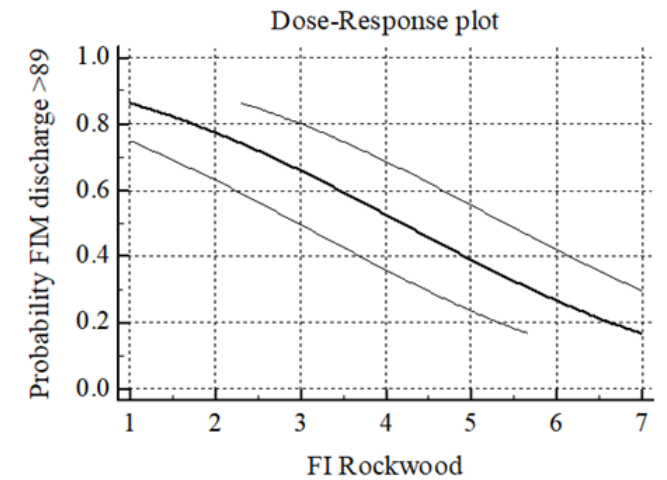

Figure 2. Dose response plots showing the probability for patients with different degrees of frailty to reach FIM discharge $\geq 90$. The lower the frailty score the higher was the probability of reaching the desired FIM threshold.

An additional analysis, by setting the total FIM discharge threshold at $\geq 85$, showen comparable results as for total FIM discharge threshold $\geq 90$.

\section{Discussion}

The question addressed was whether frailty scoring can be to predict success in rehabilitation after hip fracture. Can severity of frailty predict, better than clinical judgment, situations when rehabilitation after hip fracture is futile? A positive answer to this question would be important for clinical decision making. The short time frame needed to answer this question in rehabilitation after hip fracture offered the prospect to arrive at a clear answer. Indeed, pre-fracture frailty correlated with FIM on 
discharge, but neither frailty tool was sensitive and specific enough to predict success in rehabilitation and the length of stay in rehabilitation.

The outcome of rehabilitation is difficult to measure because several outcomes are relevant and because the relevant outcomes are affected by multiple factors in addition to treatment [26]. In practice, the use of one representative predictor of outcome, e.g., total FIM discharge or motor FIM discharge, is a necessary compromise. The validity of FIM for determining outcomes of rehabilitation is well established [27]. In a study of 117.168 Medicare beneficiaries receiving inpatient rehabilitation for hip fracture a discharge motor FIM score 58 yielded the best balance in sensitivity and specificity for discriminating patients discharged to the community from those discharged to an institution. FIM motor was equally effective as FIM total and more effective than FIM cognition to this aim, Moreover, FIM motor ratings alone were as effective as a multivariable model in discriminating patients discharged to the community from those discharged to an institution [6]. From a clinical perspective, a diagnostic tool based on a single standard measure and a defined threshold of success (e.g. discharge FIM) is more practical and meaningful than values obtained from a composite model [6], and it also is most popular [28]. Both indices, the total FIM discharge and motor FIM discharge, were used in our study.

Frailty as a predictor of short-term functional recovery after a pathological event has been investigated in diverse conditions, such as trauma, general surgery, chemotherapy, kidney transplantation, and decisions to treat hyperparathyroidism [17, 23-28]. A comprehensive literature survey investigated the association of frailty (by means of the Clinical Frailty Scale) with an outcome, mostly in hospitalized patients. Frailty was predictive in $74 \%$ of the cases, highlighting its utility in the care of older patients [29]. On the other hand, in the assessment of cardiac preoperative risk, incorporating frailty in existing models require further validation and have not been widely adopted [30,31]. Research is needed to quantify the predictive ability of validated frailty instruments in the context of different surgical procedures and existing surgical risk indices [32, 33].

Functional recovery after hip fracture was connected to the degree of frailty in previous $[5,6,28]$ as well as in the present study. The lower was the pre-fracture frailty score the higher was the probability of success in rehabilitation. Both frailty tools, the FI MDS and FI Rockwood, fitted well to the population of the present study, where the patients' physical and cognitive limitations would impede on performance of motor tests and on self-reporting. Pre-fracture FI MDS and FI Rockwood corelated strongly $(\mathrm{p}<0.0001)$ with FIM discharge score $\geq 90$ and motor FIM discharge score $>58$ (Figure 1). However, their predictive accuracy was disappointing: for FIM discharge score $\geq 90$ the best discrimination of success from failure was provided by FI MDS 12.1 with $88.5 \%$ sensitivity but merely and $53.8 \%$ specificity. Comparable results were found for FI Rockwood. Beyond commonsense used for the first triage, frailty tools performed with insufficient specificity in predicting the outcome of rehabilitation. Moreover, the frailty tools were inaccurate in predicting the pace of improvement under rehabilitation. For the disadvantaged, a longer stay in rehabilitation compensated for slower improvement (Figure 2).

In conclusion, frailty impacts the outcome of rehabilitation after proximal hip fracture. Yet, frailty tools are not useful to predict success of rehabilitation nor the necessary length of stay in rehabilitation. Translating frailty scores into clinically sensible tools to predict outcomes remains an aim for future studies.

Author Contributions: Oxana Zalyesov in the design of the study, acquisition of data; Muhamd Badarny in assessment of patients' FIM; Natalia Zaygraykin in the planning the study, review of the literature, data analysis; Jochanan E. Naschitz, conceiving and planning, data analysis, writing the manuscript.
Conflict of Interest: there were neither personal nor financial conflicts of interest to this study.

\section{References}

1. Patrick L, Psych C, Knoefel F, Gaskowski P, Rexroth D. (2001) Medical comorbidity and rehabilitation efficiency in geriatric inpatients. J Am Geriatr Soc; 49:1471-1477.

2. Lieberman D, Friger M, Lieberman D. (2006) Inpatient rehabililtation outcome after hip fracture surgery in older people patients: a prospective cohort study of 946 patients. Arch Phys Med Rehabil; 87:167-171.

3. Hershkovitz A, Kalandariov Z, Hermush V, Weiss R, Brill S. (2007) Factors affecting short-term rehabilitation outcomes of disabled older people patients with proximal hip fracture. Arch Phys Med Rehabil; 88:916-921.

4. Xu BY, Yan S, Low LL, Vasanwala FF, Low SG. (2019) Predictors of poor functional outcomes and mortality in patients with hip fracture: a systematic review. BMC Musculoskelet Disord; 20:568.

5. Inoue T, Misu S, Tanaka T, et al. (2019) Frailty defined by 19 items as a predictor of short-term functional recovery in patients with hip fracture. Injury; 50:2272-2276.

6. Wang C-Y, Graham JE, Karmarkar AM, et al. (2014) FIM motor scores for classifying community discharge after inpatient rehabilitation for hip fracture. PMR; 6:493-497.

7. Lastra-German IK, Navarrete-Reyes AP, Mejía-Domínguez NR, et al. (2019) Adjusted chemotherapy according to frailty status in older people patients with diffuse large B-cell lymphoma: Experience from a single referral center in Mexico City. Clin Lymphoma Myeloma Leuk; 19:e98-e106.

8. Seib CD, Chomsky-Higgins K, Gosnell JE, et al. (2018) Patient frailty should be used to individualize treatment decisions in primary hyperparathyroidism. World J Surg; 42:3215-3222.

9. Haugen CE, Chu NM, Ying H, et al. (2019) Frailty and access to kidney transplantation. Clin J Am Soc Nephrol; 14:576-582.

10. Kundi H, Noseworthy PA, Valsdottir LR, et al. (2020) Relation of frailty to outcomes after catheter ablation of atrial fibrillation. Am J Cardiol; 125:1317-1323.

11. Seib CD, Rochefort H, Chomsky-Higgins K, et al. (2018) Association of patient frailty with increased morbidity after common ambulatory general surgery operations. JAMA Surg; 153:160-168.

12. Hamidi M, Haddadin Z, Zeeshan M, et al. (2019) Prospective evaluation and comparison of the predictive ability of different frailty scores to predict outcomes in geriatric trauma patients. $\mathbf{J}$ Trauma Acute Care Surg; 87:1172-1180.

13. Bryant EA, Tulebaev S, Castillo-Angeles M, Moberg E, Senglaub SS, et al. (2019) Frailty identification and care pathway: an interdisciplinary approach to care for older trauma patients. J Am Coll Surg;228:852-9.e1

14. Thillainadesan J, Scott IA, Le Couteur DG. (2020) Frailty, a multisystem ageing syndrome. Age and Ageing; 49: 758-763.

15. Fried LP, Tangen CM, Walston J, et al. (2001) Frailty in Older Adults. Evidence for a Phenotype. J Gerontol Biol Sci Med Sci; 56:M146-156.

16. Kojima G. (2018) Frailty defined by FRAIL scale as a predictor of mortality: a systematic review and meta-analysis. J Am Med Dir Assoc; 19:480-483.

17. Kojima G. (2018) Quick and simple FRAIL scale predicts incident activities of daily living (ADL) and instrumental ADL (IADL) disabilities: a systematic review and meta-analysis. J Am Med Dir Assoc; 19:1063-1068. 
18. Aguayo GA, Donneau A-F, Vaillant MT, et al. (2017) Agreement between 35 Published Frailty Scores in the General Population. Am J Epidemiol; 186:420-434.

19. Jones DM, Song X, Rockwood K.Jones DM, et al. (2004) operationalizing a frailty index from a standardized comprehensive geriatric assessment. J Am Geriatr Soc; 52:19291933.

20. Theou O, Walston J, Rockwood K.Theou O, et al. (2015) Operationalizing Frailty Using the Frailty Phenotype and Deficit Accumulation Approaches. Interdiscip Top Gerontol Geriatr; 41:66-73.

21. Shaw BH, Borrel D, Sabbaghan K, et al. (2019) Relationships between orthostatic hypotension, frailty, falling and mortality in older people care home residents. BMC Geriatr; 19:80.

22. Rockwood K, Song X, MacKnight C, et al. (2005) A global clinical measure of fitness and frailty in older people people. CMAJ; 173:489-495.

23. Hutchinson AM, Milke DL, Maisey S, et al. (2012) The Resident Assessment Instrument-Minimum Data Set 2. Psychiatr Nurses Assoc; 18(2 SRC-GoogleScholar FG-0):1472-6963.

24. Folstein MF, Folstein SE, McHugh PR. "Mini-mental state". (1975) A practical method for grading the cognitive state of patients for the clinician. J Psychiatr Res; 12:189-198.

25. Linacre JM, Heinemann JW, Wright BD, Granger CV, Hamilton BB. (1994) The structure and stability of the functional independence measure. Arch Phys Med Rehabil; 75: 127-132.
26. Ottenbacher KJ, Hsu Y, Granger CV, Fiedler RC. (1996) The reliability of the functional independence measure: a quantitative review. Arch Phys Med Rehabil; 77:1226-1232.

27. Skinner A, Turner-Stokes L. (2006) The use of standardized outcome measures in rehabilitation centers in the UK. Clin Rehabil; 20:609-615.

28. Di Monaco M, Castiglioni C, De Toma E, Gardin L, Giordano S, Tappero R. (2015) Handgrip strength is an independent predictor of functional outcome in hip-fracture women: A prospective study with 6-month follow-up. Medicine (United States); 94:e542.

29. Church S, Rogers E, Rockwood K, Theou O. (2020) A scoping review of the Clinical Frailty Scale. BMC Geriatr; 20:393.

30. Sepehri A, Beggs T, Hassan A, Rigatto C, Shaw-Daigle C, Tangri $\mathrm{N}$, et al. (2014) The impact of frailty on outcomes after cardiac surgery: a systematic review. J Thorac Cardiovasc Surg; 148:3110-117.

31. Bagnall NM, Faiz O, Darzi A, Athanasiou T. (2013) What is the utility of preoperative frailty assessment for risk stratification in cardiac surgery? Interact Cardiovasc Thorac Surg; 17:398-402.

32. Khatry K, Peel NM, Gray LC, Hubbard RE. (2018) The utility of the Frailty Index in clinical decision making. J Frailty Aging; 7:138-141.

33. Ko FC. (2019) Preoperative frailty evaluation: a promising riskstratification tool in older adults undergoing general surgery. Clin Ther; 41:387-399.

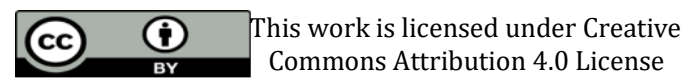

To Submit Your Article Click Here: Submit Manuscript

DOI: $10.31579 / 2693-4779 / 049$
Ready to submit your research? Choose Auctores and benefit from:

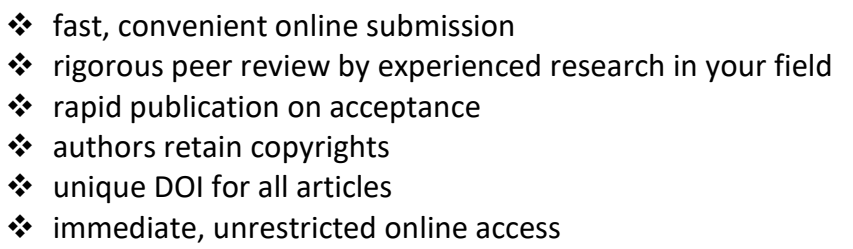

At Auctores, research is always in progress.

Learn more www.auctoresonline.org/journals/clinical-research-andclinical-trials 